\title{
ON QUATERNIONIC JAMES NUMBERS AND ALMOST-QUATERNION SUBSTRUCTURES \\ ON THE SPHERE
}

\author{
TURGUT ONDER
}

\begin{abstract}
ABSTRACr. In this paper a theorem about the relation between the divisibility of orders of obstructions to cross sectioning symplectic Stiefel manifolds and quaternionic James numbers is proved. As an application of this, the existence problem of almost-quaternion $k$-substructures on the sphere $S^{n}$ is solved for all $n$ and $k$ except for the case $n=4 m-3, k=m-1$ for some $m \geqslant 1$.
\end{abstract}

1. Introduction. Let $S p(n)$ be the symplectic group and $X_{n, k}=S p(n) / S p(n-k)$ the symplectic Stiefel manifold. Consider the following exact homotopy sequence of the natural projection onto $X_{n, 1}=S^{4 n-1}$ :

$$
\rightarrow \pi_{4 n-1} X_{n, k} \stackrel{v_{\#}}{\rightarrow} \pi_{4 n-1}\left(S^{4 n-1}\right) \stackrel{\delta}{\rightarrow} \pi_{4 n-2} X_{n-1, k-1} \rightarrow \cdots .
$$

Let $\left[\iota_{4 n-1}\right]$ denote a generator of $\pi_{4 n-1}\left(S^{4 n-1}\right)$. It is immediate that $X_{n, k}$ admits a cross section if and only if $\delta\left(\left[\iota_{4 n-1}\right]\right)=0$. In a paper of I. M. James [5], the order of $\delta\left(\left[\iota_{4_{n-1}}\right]\right)$ is denoted by $X\{n, k\}$ and several properties of these numbers are introduced.

In [9] F. Sigrist and U. Suter prove that for $k>2$, the symplectic Stiefel fibering $v: X_{n, k} \rightarrow X_{n, 1}=S^{4 n-1}$ has a cross section if and only if $n$ is a multiple of the integer $d_{k}$, called quaternionic James number. This number is defined by its decomposition into prime powers as follows.

$$
\begin{aligned}
& v_{2}\left(d_{k}\right)=\max _{s}\left(2 k-1,2 s+v_{2}(s)\right), \quad 1 \leqslant s \leqslant k-1, \\
& v_{p}\left(d_{k}\right)=\max _{t}\left(t+v_{p}(t)\right), \quad 1 \leqslant t \leqslant\left[\frac{2 k-1}{p-1}\right], p \text { odd }<2 k, \\
& v_{p}\left(d_{k}\right)=0, \quad p \text { odd }>2 k .
\end{aligned}
$$

(Here $v_{p}(q)$ is the exponent of the prime $p$ in the prime decomposition of $q$. Throughout the rest of the paper we will adopt the notation $\alpha_{p}(q)=p^{v_{p}(q)}$ of [8].)

On the other hand, in [6] I. M. James proves that $X_{n, 2} \rightarrow S^{4 n-1}$ has a cross section if and only if $24 \mid n$. Let us define $d_{2}$ to be 24 , and $d_{1}$ to be 1 . Thus the number $d_{k}$ is defined for all $k \geqslant 1$.

Received by the editors July 23, 1981 and, in revised form, December 24, 1981.

1980 Mathematics Subject Classification. Primary 55S40; Secondary 53C15.

Key words and phrases. Sectioning fiber spaces and bundles, almost-complex, contact, symplectic, almost product structures. 
In $\$ 2$ we prove the following theorem, which is a quaternionic analogue of Theorem 1 of F. Sigrist in [8].

THEOREM 1.1. (i) If $n$ is a multiple of $\alpha_{p}\left(d_{k}\right)=p^{v_{p}\left(d_{k}\right)}$ for $k \geqslant 1$, p prime, then $X\{n, k\}$ is prime to $p$.

(ii) Suppose $X\{n, k\}$ is prime to $p$. Then $\alpha_{p}\left(d_{k}\right) \mid n$ unless all three of the following are true.

(a) $p=2$.

(b) $n<2 k$.

(c) $k$ cannot be written as $s+m 2^{s-1}$ for positive integers $s$ and $m$.

Under these three conditions, the weaker statement $\alpha_{p}\left(d_{k}\right) \mid 2 n$ is true.

REMARK. In this theorem, the second statement of (ii) may well be improved, for there is no example known to the author with $X\{n, k\}$ prime to 2 and $\alpha_{2}\left(d_{k}\right) \nmid n$.

The existence problem of almost-complex substructures on the sphere was completely solved by İ. Dibağ in 1976 (see [3]). Using his methods and Theorem 1.1 above, we investigate for which values of $n$ and $k$ there is an almost-quaternion $k$-substructure on the sphere $S^{n}$ (see $\S 3$ ).

Let $M$ be an orientable $n$-manifold. By an almost-quaternion $k$-substructure on $M$ we mean a reduction of the structure group of $T(M)$ from $S O(n)$ to $S p(k) \times$ $S O(n-4 k)$. Thus, on $S^{n}$ there exists an almost-quaternion $k$-substructure if and only if the fibration

$$
S O(n) / S p(k) \times S O(n-4 k) \rightarrow S O(n+1) / S p(k) \times S O(n-4 k) \rightarrow S^{n}
$$

has a cross section.

The main result of $\S 3$ is the following theorem.

THEOREM 1.2. (i) There is no almost-quaternion substructure on $S^{n}$ if $n$ is even.

(ii) There is no almost-quaternion $k$-substructure on $S^{n}$ if $n=4 m-3$ for some $m$ and $k \neq m-1$.

(iii) For $n=4 m-1, k<n / 8, S^{n}$ admits an almost-quaternion $k$-substructure if and only if $c_{4 k} \mid 4 m$. (Here $c_{k}=2^{e_{k}}$ where $e_{k}=$ the number of integers $t, 0<t \leqslant k$, $t=0,1,2,4(\bmod 8)$.)

(iv) For $n=4 m-1, k>n / 8$ and $m \geqslant 2, S^{4 m-1}$ admits an almost-quaternion $k$-substructure if and only if $\alpha_{2}\left(d_{m-k}\right) \mid m$.

REMARK. (1) In part (iii), $c_{k}$ is just the dimension of the irreducible (ungraded) representation module for the Clifford algebra $C_{k}$.

(2) Our methods do not say much for the existence of almost-quaternion $(m-1)$ substructures on $S^{4 m-3}$. This requires further study.

2. Orders of obstructions and quaternionic James numbers. Our aim in this section is to prove Theorem 1.1. The main references for this section are [2, 5 and 8$]$.

First we introduce the following terminology.

Let $P$ be a set of primes and $P^{\prime}$ the complementary set of primes. We will call a space $X$ with a base point reducible $(\bmod P)$ if $\tilde{H}_{i}(X) \cong \tilde{H}_{i}\left(S^{n}\right)$ for $i \geqslant n$ and if there exists a map $f: S^{n} \rightarrow X$ such that $f_{*}: \tilde{H}_{i}\left(S^{n}\right) \rightarrow \tilde{H}_{i}(X)$ is multiplication by an 
integer $\lambda$ prime to the primes in $P^{\prime}$. Similarly we will call a space $X$ with a base point coreducible $(\bmod P)$ if $\tilde{H}_{i}\left(S^{n}\right) \cong \tilde{H}_{i}(X)$ for $i \leqslant n$, and if there exists a map $g$ : $X \rightarrow S^{n}$ such that $g^{*}: \tilde{H}^{i}\left(S^{n}\right) \rightarrow \tilde{H}^{i}(X)$ is multiplication by an integer $\lambda$ for $i \leqslant n$, where $\lambda$ is prime to the primes in $P^{\prime}$. We will say that $X$ is $S$-reducible $(\bmod P$ ) or $S$-coreducible $(\bmod P)$ if some suspension of $X$ is reducible $(\bmod P)$ or coreducible $(\bmod P)$ respectively.

It can be easily proved that $X$ is $S$-reducible $(\bmod P$ ) if and only if its $S$-dual is $S$-coreducible $(\bmod P)$. The proof of this is the same as the proof of the analogous result about ordinary $S$-reducibility (e.g. see the proof of Theorem 8.4 in Chapter 15 of [4]).

Next, we modify the proofs of Proposition 2.8 and Theorem 6.5 of [2] to get the analogous results for $(\bmod P)$ case. The main difference is that the following theorem of [7] is used instead of Dold's results in the proof of Proposition 2.8 in [2] (see Theorem 2.1 in [7]).

Proposition 2.1. Let $\alpha$ and $\beta$ be $S^{k}$-fibrations over a finitely-dominated $\mathrm{CW}$ complex, $k$ large. Then the following are equivalent.

(a) The Stiefel-Whitney classes $w_{1}(\alpha)$ and $w_{1}(\beta)$ are equal, and there is an integer $d$, prime to the primes in $P$, such that $d \alpha$ is fibre-homotopy equivalent to $d \beta$.

(b) There is a map $h: \alpha \rightarrow \beta$ (covering the identity) of degree $d^{\prime}$ on each fibre, where $d^{\prime}$ is prime to the elements of $P$.

THEOREM 2.2. Let $\alpha$ be a real vector bundle over a connected space $X$. If the Thom complex $T(\alpha)$ is $S$-coreducible $(\bmod P)$, then there exists an integer $\mu$ prime to the primes in $P^{\prime}$ such that $\mu J(\alpha)=0$.

Proof. We consider $m$ so large that (1) $\eta=\alpha \oplus \theta^{m}$ has the property that $T(\eta)=T\left(\alpha \oplus \theta^{m}\right)=S^{m}(T(\alpha))$ is coreducible $(\bmod P)$; (2) Proposition 2.1 holds; (3) we have the commutative diagram (which exists for large $n$; see [4, Chapter 15, Theorem 7.7])

$$
\begin{array}{ccc}
{\left[S(\eta), S^{n-1}\right]} & \rightarrow & {\left[D(\eta) / S(\eta), S^{n}\right]} \\
\downarrow \theta & & \downarrow \phi \\
{\left[S\left(\eta_{x}\right), S^{n-1}\right]} & \rightarrow & {\left[D\left(\eta_{x}\right) / S\left(\eta_{x}\right), S^{n}\right]}
\end{array}
$$

where the horizontal maps are isomorphisms. Here $D(\eta)$ and $S(\eta)$ denotes associated unit disc bundle and associated sphere bundle of $\eta$, for some Riemannian metric on $\eta$ and $T(\eta)$ is just $D(\eta) / S(\eta)$.

Now if $T(\eta)$ is coreducible $(\bmod P)$ then there exists a map $g: D(\eta) / S(\eta) \rightarrow S^{n}$ which induces multiplication by $\lambda$ between the cohomology groups for $i \leqslant n$. Since the inclusion $\left(D\left(\eta_{x}\right), S\left(\eta_{x}\right)\right) \rightarrow(D(\eta), S(\eta))$ induces isomorphisms of cohomology groups for $0 \leqslant r \leqslant n$ (e.g. see 7.5 of Chapter 15 of [4]), this is equivalent to saying that the image of $[g]$ under $\phi$ is the class of a map of degree $\lambda$ between $D\left(\eta_{x}\right) / S\left(\eta_{x}\right)$ and $S^{n}$. Then by Proposition 2.1, there exists an integer $\mu$ such that $\mu J(\eta)=0$.

Next let $Q_{n, k}$ be the quaternionic stunted quasi-projective space as defined in $\$ 5$ of [2]. We recall that there exists a bundle $\zeta$ (see $\S 4$ of [2] for the definition) such that 
$Q_{n, k}$ is homeomorphic to $T((n-k) \xi \oplus \zeta)$. Its $S$-dual is $T(-[(n-k) \xi \oplus \zeta]-\tau)$, where $\xi$ is the underlying real vector bundle of the canonical quaternionic line bundle over $H P^{k-1}$, and $\tau$ is the tangent bundle of $H P^{k-1}$. We then get the mod $P$-analogue of Theorem 6.5 in [2].

TheOREM 2.3. Let $n \geqslant 2 k$. Then if $X\{n, k\}$ is prime to $p$ there exists an integer $\mu$ prime to $p$ such that $\mu n J(\xi)=0$.

Proof. Let $v$ and $\delta$ be as in the introduction. By definition

$$
X\{n, k\}=\operatorname{order} \delta\left(\left[\iota_{4 n-1}\right]\right) .
$$

If $X\{n, k\}$ is prime to $p$, then there exists $f: S^{4 n-1} \rightarrow X_{n, k}$ such that $v \circ f: S^{4 n-1} \rightarrow$ $S^{4 n-1}$ is of degree $\lambda$ prime to $p$. But by 8.2 of [6], for $n \geqslant 2 k$ we have $\pi_{4 n-1}\left(Q_{n, k}\right) \cong$ $\pi_{4 n-1}\left(X_{n, k}\right)$. Therefore if $X\{n, k\}$ is prime to $p$, then $Q_{n, k}$ is reducible $\left(\bmod \{p\}^{\prime}\right)$; but this is true if and only if its $S$-dual $T(-[(n-k) \xi \oplus \zeta]-\tau)$ is $S$-coreducible $\left(\bmod \{p\}^{\prime}\right)$. Therefore if $X\{n, k\}$ is prime to $p$ then $\mu J(-[(n-k) \xi \oplus \zeta]-\tau)=0$ for an integer $\mu$ prime to $p$. Using the relation $\tau \oplus 1 \oplus \zeta \cong k \xi$ (Lemma 4.5 of [2]), we get $\mu n J(\xi)=0$.

Proof of Theorem 1.1. (i) Assume $\alpha_{p}\left(d_{k}\right) \mid n$. By the proof of Theorem 1 in [8], $X\{n, k\}$ is prime to $p$ if $n$ is a multiple of $\alpha_{p}\left(d_{k}\right)$ for $n(p-1)>p(k-1)$. (F. Sigrist proves this for the complex case in [8], but the same argument is valid for $X\{n, k\}$ also.) So it suffices to show that

$$
\alpha_{p}\left(d_{k}\right)(p-1)>p(k-1) \text { for } 2 \leqslant p<2 k .
$$

But for $2 k>2$, we know the following inequalities, again from the proof of Theorem 1 in [8];

$$
\begin{aligned}
2 & \leqslant p \leqslant \frac{2 k+1}{2} \Rightarrow \alpha_{p}\left(b_{2 k}\right)(p-1)>p(2 k-1), \\
\frac{2 k+1}{2} & <p<2 k \Rightarrow 2 \alpha_{p}\left(b_{2 k}\right)(p-1)>p(2 k-1),
\end{aligned}
$$

where $b_{2 k}$ denotes the complex James number. The result follows because $d_{k}$ is either $b_{2 k}$ or $b_{2 k} / 2$ by Proposition 4.0 of [9].

(ii) If $X\{n, k\}$ is prime to $p$ and $n \geqslant 2 k$, then by Theorem 2.3 there exists an integer $\mu$ prime to $p$ such that $\mu n J(\xi)=0$. Hence $d_{k} \mid \mu n$ and therefore $\alpha_{p}\left(d_{k}\right) \mid n$.

So we assume $n<2 k$. Let $W\{n, k\}$ denote the order of the obstruction to cross sectioning the complex Stiefel fibering $W_{n, k} \rightarrow S^{2 n-1}$. The remaining statements follow because (1) $W\{2 n, 2 k\}$ divides $X\{n, k\}$ (by Theorem 2.2 of [5]); (2) by the results of [8], $W\{2 n, 2 k\}$ is prime to $p$ if and only if $\alpha_{p}\left(b_{2 k}\right)$ divides $2 n$; (3) we know that $d_{k}=b_{2 k} / 2$ whenever $k=s+m 2^{s-1}$ for positive integers $s$ and $m, d_{k}=b_{2 k}$ otherwise (see Theorem 4.1 of [9]).

3. Almost-quaternion substructures on spheres. Our aim in this section is to prove Theorem 1.2. The general reference for this section is İ. Dibağ's paper [3]. We used the same technique as he used to obtain analogous results for almost-complex substructures. 
Proof of Theorem 1.2. (i) Let $n$ be even. Then there cannot be an almostquaternion substructure on $S^{n}$, because the existence of an almost-quaternion substructure implies the existence of an almost-complex substructure, and it is proved in [3] that the only almost-complex substructures on even dimensional spheres are the almost-complex structures on $S^{2}$ and $S^{6}$.

(ii) Let $n=4 m-3$. Then by Theorem 27.18 of [10], $S^{4 m-3}$ does not admit a continuous field of $4 k$-planes for $2 \leqslant 4 k \leqslant n-2$. So $S^{4 m-3}$ can admit a continuous field of $4 k$-planes only if $4 k=n-1=4(m-1)$.

(iii) Let $k<(4 m-1) / 8$. By 27.16 of [10], $S^{n}$ admits a continuous field of tangent $4 k$-planes if and only if it admits a continuous $4 k$-field. But by [1], $S^{4 m-1}$ admits a field of $4 k$-frames if and only if $c_{4 k} \mid 4 m$.

(iv) To prove this part, we prove the following lemmas first.

LEMMA 3.1. For $k>(4 m-1) / 8, m>2$, there exists an almost-quaternion $k$-substructure on $S^{4 m-1}$ if and only if the fibering

$$
S O(4 m-1) / S p(k) \rightarrow S O(4 m) / S p(k) \rightarrow S^{4 m-1}
$$

has a cross section.

Proof. The proof of this lemma is precisely the same as 3.2 of [3]. Only $n$ should be replaced by $2 m, s$ by $2 k$ and $\pi_{2 n-2} U(n)$ by $\pi_{4 m-2} S p(m)$.

LEMMA 3.2. Let $u: S O(4 m-1) \rightarrow S O(4 m-1) / S p(m-1) \cong S O(4 m) / S p(m)$ be the natural projection and

$$
\partial_{u}: \pi_{4 m-1} S O(4 m-1) / S p(m-1) \rightarrow \pi_{4 m-2} S p(m-1)
$$

be the boundary homomorphism in the homotopy exact sequence of $u$. Then the image of $\partial_{u}$ is the subgroup generated by $2 x_{m, m}$ where $x_{m, m}$ is the obstruction to cross sectioning the fibration

$$
S p(m-1) \rightarrow S p(m) \rightarrow S^{4 m-1}
$$

PROOF. We make the necessary changes in the proof of 4.2 of [3].

By taking $H=S O(4 m-1), K=S p(m), G=H K=K H=S O(4 m), H \cap K=$ $S p(m-1)$ in Corollary 4.1 of [3], we obtain the following anticommutative square.

$$
\begin{aligned}
& \pi_{4 m-1} S O(4 m) \stackrel{r_{\#}}{\rightarrow} \pi_{4 m-1} S O(4 m) / S p(m) \stackrel{\partial_{r}}{\rightarrow} \pi_{4 m-2} S p(m) \\
& p_{\#} \downarrow \\
& \pi_{4 m-1} S^{4 m-1} \stackrel{\partial_{q}}{\rightarrow} \quad \pi_{4 m-2} S p(m-1)
\end{aligned}
$$

The upper row is part of the exact homotopy sequence of the projection $S O(4 \mathrm{~m}) \rightarrow$ $S O(4 m) / S p(m)$, the lower row is the exact homotopy sequence of the projection $S p(m-1) \rightarrow S p(m) \rightarrow S^{4 m-1}$, and $p$ is the fibering $S O(4 m-1) \rightarrow S O(4 m) \rightarrow$ $S^{4 m-1}$.

Since $\pi_{4 m-2} S p(m)=0, r_{\#}$ is an epimorphism and hence we have $\operatorname{Im} \partial_{u}=$ $\operatorname{Im}\left(\partial_{u} r_{\#}\right)=\operatorname{Im}\left(\partial_{q} p_{\#}\right)$. But if $\left[\iota_{4 m-1}\right]$ is the generator of $\pi_{4 m-1}\left(S^{4 m-1}\right)$, then 
$2\left[\iota_{4 m-1}\right] \in \operatorname{Im} p_{\#}$ by 23.4 of $[10]$ and $x_{m, m}=\partial_{q}\left(\left[\iota_{4 m-1}\right]\right)$ by definition. Therefore $\operatorname{Im} \partial_{u}$ is generated by $2 x_{m, m}$.

Notation. The obstruction to finding a section for the fibration $S p(m) / S p(k) \rightarrow$ $S p(m) / S p(m-1)=S^{4 m-1}$ will be written as $x_{m, m-k}$.

LEMMA 3.3. For $m>2$, the fibration

$$
S O(4 m-1) / S p(k) \rightarrow S O(4 m) / S p(k) \rightarrow S^{4 m-1}
$$

admits a cross section if and only if $x_{m, m-k}$ is of odd order.

Proof. Consider the diagram

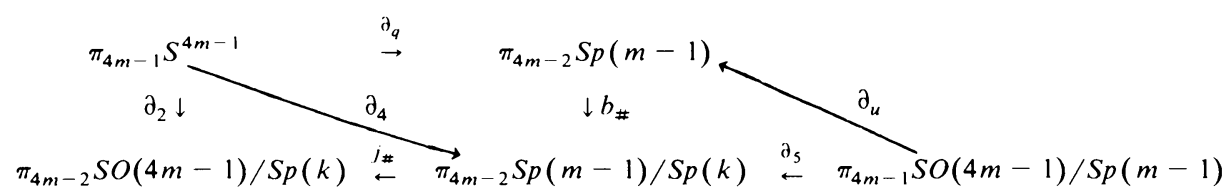

Now the fibration $S O(4 m) / S p(k) \rightarrow S^{4 m-1}$ has a cross section if and only if $\partial_{2}=0$ and hence iff $j_{\#} \partial_{4}=j_{\#}\left(b_{\#} \partial_{q}\right)=0$. By exactness of the bottom row, this is true iff $\operatorname{Im}\left(b_{\#} \partial_{q}\right)$ is in $\operatorname{Im} \partial_{5}=\operatorname{Im}\left(b_{\#} \partial_{u}\right)$. By naturality of obstructions, $b_{\#} \partial_{q}\left(\left[\iota_{4 m-1}\right]\right)$ $=x_{m, m-k}$. On the other hand, by Lemma 3.2 this element is in $\operatorname{Im}\left(b_{\#} \partial_{u}\right)$ iff it lies in the subgroup generated by $2 x_{m, m-k}$; in other words, if and only if $x_{m, m-k}$ has odd order.

For the case $m>2$ and $k>(4 m-1) / 8$, the proof of (iv) follows from Lemmas 3.1 and 3.3, and Theorem 1.1. Finally, for the case $m=2$ we have $4 m-1=7$. But $S^{7}$ is parallelisible, so it obviously admits a 1 -substructure. This completes the proof of Theorem 1.2.

ADDED IN PROOF. After the submission of this paper we have proved that $\alpha_{p}\left(d_{k}\right) \mid n$ holds under conditions (a), (b), (c) of Theorem 1.1(ii) also.

\section{REFERENCES}

l. J. F. Adams, Vector fields on spheres, Ann. of Math. (2) 75 (1962), 603-632. MR $25 \# 2614$.

2. M. F. Atiyah, Thom complexes, Proc. London Math. Soc. (3) 11 (1961), 291-310. MR 24 \#1727.

3. İ. Dibağ, Almost-complex substructures on the sphere, Proc. Amer. Math. Soc. 51 (1976).

4. D. Husemoller, Fibre bundles, Springer-Verlag, Berlin and New York, 1966.

5. I. M. James, Cross sections of Stiefel manifolds, Proc. London Math. Soc. (3) 8 (1958), 536-547.

6. Spaces associated with Stiefel manifolds, Proc. London Math. Soc. (3) 9 (1959), 115-140.

7. P. J. Kahn, Mixing homotopy types of manifolds, Topology 14 (1975), 203-216.

8. F. Sigrist, Sur les nombres de James des variétés Stiefel complexes, Illinois J. Math. 13 (1969), 198-201. MR 40 \#2078.

9. F. Sigrist and U. Suter, Cross sections of symplectic Stiefel manifolds, Trans. Amer. Math. Soc. 184 (1973), 247-259.

10. N. E. Steenrod, The topology of fibre bundles, Princeton Univ. Press, Princeton, N. J., 1951. MR 12, 522.

Department of Mathematics, Middle East Technical University, Ankara, Turkey 